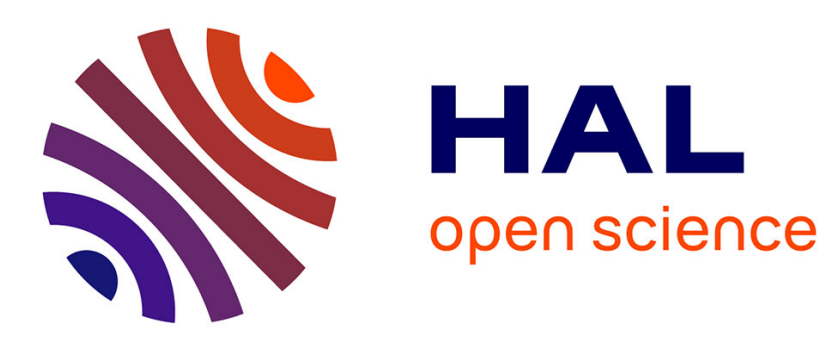

\title{
Elastic Shape Models for Face Analysis Using Curvilinear Coordinates
}

Anuj Srivastava, Chafik Samir, Shantanu H. Joshi, Mohamed Daoudi

\section{To cite this version:}

Anuj Srivastava, Chafik Samir, Shantanu H. Joshi, Mohamed Daoudi. Elastic Shape Models for Face Analysis Using Curvilinear Coordinates. Journal of Mathematical Imaging and Vision, 2009, 33 (2), pp.253-265. 10.1007/s10851-008-0073-6 . hal-00665940

\section{HAL Id: hal-00665940 https://hal.science/hal-00665940}

Submitted on 4 Feb 2012

HAL is a multi-disciplinary open access archive for the deposit and dissemination of scientific research documents, whether they are published or not. The documents may come from teaching and research institutions in France or abroad, or from public or private research centers.
L'archive ouverte pluridisciplinaire HAL, est destinée au dépôt et à la diffusion de documents scientifiques de niveau recherche, publiés ou non, émanant des établissements d'enseignement et de recherche français ou étrangers, des laboratoires publics ou privés. 


\title{
Elastic Shape Models for Face Analysis Using Curvilinear Coordinates
}

\author{
A. Srivastava ${ }^{1}$, C. Samir ${ }^{2}$, S. H. Joshi ${ }^{3}$, M. Daoudi ${ }^{2}$ \\ 1 Department of Statistics, Florida State University, Tallahassee, FL 32306, USA \\ 2 GET/Telecom Lille1, LIFL (UMR USTL/CNRS 8022), France \\ 3 Department of Electrical Engineering, Florida State University, Tallahassee, FL 32306, USA
}

The date of receipt and acceptance will be inserted by the editor

\begin{abstract}
This paper studies the problem of analyzing variability in shapes of facial surfaces using a Riemannian framework, a fundamental approach that allows for joint matchings, comparisons, and deformations of faces under a chosen metric. The starting point is to impose a curvilinear coordinate system, named the Darcyan coordinate system, on facial surfaces; it is based on the level curves of the surface distance function measured from the tip of the nose. Each facial surface is now represented as an indexed collection of these level curves. The task of finding optimal deformations, or geodesic paths, between facial surfaces reduces to that of finding geodesics between level curves, which is accomplished using the theory of elastic shape analysis of 3D curves. Elastic framework allows for nonlinear matching between curves and between points across curves. The resulting geodesics provide optimal elastic deformations between faces and an elastic metric for comparing facial shapes. We demonstrate this idea using examples from FSU face database.
\end{abstract}

\section{Introduction}

There is an increasing interest in analyzing shapes of facial surfaces with many applications including biometrics, facial surgery, video communications, and 3D animation. This interest is fuelled by the advent of cheaper and lighter scanners that can provide high-resolution measurements of both geometry and texture of human facial surfaces. One general goal here is to develop computational tools for analyzing 3D face data. In particular, one is interested in comparing the shapes of facial surfaces. Such a tool can be used to recognize human beings according to their facial shapes, to measure changes in a facial shape due to a surgery, or to study/capture the variations in facial shapes during conversations and expressions of emotions. Additionally, a subproblem may be to find an optimal deformation of one surface into another, under some chosen criterion. These deformations can be useful in defining summary statistics of a collection of faces. For example, one can compute an average of faces of different people, under different facial expressions. Efficient tools for understanding and studying variability in facial shapes are of great importance in our biometric-oriented society. Accordingly, the main theme of this paper is to develop a computational framework for analyzing shapes of facial surfaces.

\subsection{Major Issues in Surface Analysis}

What are the difficulties in performing standard shape analysis on the face data? The main issue is that there is no canonical coordinate system to represent and study the geometry of a nonlinear surface, such as a face. To highlight this issue, contrast the geometry of a surface with that of a curve. The points along a curve have a fixed ordering which allows us to impose a coordinate system for studying shapes of curves. Although the parametrization for a curve may not be unique, the task of analyzing shapes of curves modulo all possible parameterizations is still tractable. More specifically, the space of all possible parameterizations 
of a curve is the set of all one-dimensional diffeomorphisms, and there exist efficient algorithms (such as the dynamic programming algorithm) for optimally matching curves over all parameterizations. For surfaces, there is no natural ordering of points and, thus, no natural way of parameterizing them. Thus, the goal of analyzing shapes of surfaces, modulo all possible re-parameterizations, becomes quite difficult and leads to enormous computational challenges.

\subsection{Past Work}

Past literature has tackled this fundamental issue with a varying degree of success, depending on the ultimate application. If we divide the main tasks in facial shape analysis into representing, matching, comparing, and deforming, several past papers that have addressed these individual tasks.

1. Surface Representations \& Feature Comparisons: For comparing faces, a common theme in this literature has been to represent facial surfaces by certain geometrical features, such as the height function [16], convex regions, areas with high curvatures [15], saddle points, etc. These methods provide reasonable results in classification and clustering of faces, but they do not study deformations nor develop statistical analysis of shapes. Additionally, although some of these features are intuitively meaningful, their computations, e.g. those involving second derivatives, may be susceptible to observation noise. Other approaches, such as those based on shape distribution [33] and conformal geometry [40], have also been proposed for matching surfaces. Beumier and Acheroy [2] have used shapes of facial profiles to compare faces.

Another commonly used approach, that relates to our paper, is to define smooth functions, more specifically Morse functions, on surfaces and to use the level curves of these functions for defining coordinates on surfaces. Samir et al. [34] used the level curves of the height function to define and study shapes of facial curves. Bronstein et al. [4] used the geodesic distances on facial surfaces to perform surface matching and comparisons. This distance has also been used by many other authors, including Gupta [14] and Samir et al. [36], etc. Mpiperis et al. [32] have used this representation to match features across faces.

2. Matching Facial Surfaces: There are two types of matching algorithms: rigid and non-rigid. Rigid surface matching, often viewed as a matching of two point clouds in $\mathbb{R}^{3}$, is performed using the Iterative Closest Point (ICP) algorithm (see for example [1]) or its modifications. For non-rigid matching, several ideas have been proposed. Lu et al. [26,27] have used thin plate splines to perform non-rigid matching of faces. Chui and Rangarajan [8] have presented a robust algorithm for non-rigid deformation and registration of point sets. Chang et al. [6] have suggested restricting to some prominent parts of faces, such as the nose region, for performing the matching. One idea that is often used in medical image analysis is the use of mutual information to register points across high-dimensional images. This idea has been applied to matching of facial surfaces by Wang et al. [41]. However, this approach is often applied to matching using statistics of pixel values in images rather than geometric properties of a surface.

Bronstein et al. [3] study the problem of matching points across surfaces in an isometric manner. Here the task of matching surfaces has been accomplished by minimizing the Gromov-Hausdorff distance using multidimensional scaling [28]. A Riemannian analysis of facial surfaces developed here is different from Bonstein et al. in that the matching and deformation energies are based on lengths of geodesic paths (under the chosen Riemannian metric) and not a global distance like Gromov-Hausdorff. For shape analysis of planar shapes using Hausdorff distances, Charpiat et al. [7] have presented a Riemannian framework, but such a connection for surfaces remains to be presented.

3. Deforming Facial Surfaces: In recent years there has been focus on deforming surfaces, one into another, under a chosen criterion. Grenander's deformable template theory [11] has been successfully applied to studying shapes of anatomical parts using medical images [30,12]. The set of non-rigid deformations can be subdivided into linear and nonlinear deformations. Nonlinear deformations imply local stretching, compression, and bending of surfaces to match each other, and are also referred to as elastic deformations. Earlier attempts at elastic matching utilized graphs based on texture images of faces [42,24]. Kakadiaris et al. [19] utilize an annotated face model to study geometrical variability across faces. Glaunes et al. [9] have studied diffeomorphic matching of points sets in two and three-dimensions. Litke et al. [25] take an interesting approach by representing surfaces as 2D images, using arbitrary parameterizations, and matching of surfaces is accomplished by matching the corresponding 2D image coordinates. The 
cost function for matching includes distortions introduced due to parameterizations, deformations, and features mismatch. A recent paper by Kilian et al. [21] describes a numerical approach to computing geodesic paths between registered triangulated surfaces using Euclidean metrics; the invariance of this approach to arbitrary parameterizations of surfaces was not discussed.

\subsection{Our Approach}

We present a unified Riemannian framework that provides optimal matching, comparisons and deformations of faces using a single elastic metric. The following items highlight the contribution of this paper.

1. Darcyan Curvilinear Coordinate System: Rather than allowing arbitrary parameterizations of facial surfaces, as in [25], we choose a specific coordinate system that is natural for analyzing shapes of facial surfaces. We use the geodesic distance function from a fixed reference point to define this system. Being intrinsic to the surface, it is invariant to rigid motions (rotations and translations). The fact that it deforms with the changes in facial expressions provides a possibility of countering variability in facial shapes induced by changes in facial expressions, as noted by several earlier papers $[4,14]$. We will denote the coordinate system by $\Xi$ and points in $\Xi$ by $\xi$. The level sets of coordinates $\xi_{1}$ or $\xi_{2}$ are curves that vary in shapes from face to face. This fact is exploited in this paper to characterize shapes of facial surfaces. Such a curvilinear coordinate system has been proposed earlier for use in medical image analysis by Grenander et al. [13] who referred to it as the Darcyan coordinate system, after the famous shape analyst D'Arcy Thompson.

2. Elastic Matching: An important requirement of a face analysis system is the non-rigid or elastic matching of points across faces. We claim that the task of elastic matching becomes relatively simpler in a Darcyan coordinate system. Let $\Xi$ be the Darcyan coordinate system associated with one face and $\Xi^{\prime}$ with another. The main task in shape matching is to find a mapping $\phi: \Xi \mapsto \Xi^{\prime}$ that is a diffeomorphism (smooth and invertible, with a smooth inverse). If $\phi$ s are considered random, then shapes should be compared by integrating over all possible $\phi$ s.

$$
d\left(S_{1}, S_{2}\right)=\int_{\phi} d_{\kappa}\left(\kappa^{1}(\xi), \kappa^{2}(\phi(\xi))\right) P(\phi) d \phi,
$$

where

$-\kappa$ is some geometric descriptor being used to compare the surfaces,

$-d_{\kappa}$ is an appropriate distance on the space of that descriptor, and

$-P(\phi)$ is the probability density on $\phi$.

However, in practice one uses an optimal $\phi$ under a pre-determined criterion, to match shapes. This requires solving an optimization problem over the set of all mapping $\phi$ s.

$$
d\left(S_{1}, S_{2}\right) \approx d_{\kappa}\left(\kappa^{1}(\xi), \kappa^{2}\left(\phi^{*}(\xi)\right)\right), \text { where } \phi^{*}=\underset{\phi}{\operatorname{argmin}} d_{\kappa}\left(\kappa^{1}(\xi), \kappa^{2}(\phi(\xi))\right) .
$$

The advantage of a Darcyan coordinate system is that the search for this optimal $\phi$ can be constrained further. While in past papers, e.g. Litke et al. [25], surface matching has been studied as a full 2D matching problem, in our approach it is restricted to two 1D matching problems. Here, the mapping $\phi\left(\xi_{1}, \xi_{2}\right)$ decomposes into the form $\phi_{1}\left(\xi_{1}\right) \phi_{2}\left(\xi_{2} ; \xi_{1}\right)$. The first function $\phi_{1}$ simply matches the level curves of $\xi_{1}$ on $S_{1}$ with the level curves of $\xi_{1}^{\prime}$ on $S_{2}$. Then, the second function $\phi_{2}$ matches points across the curves previously matched using $\phi_{1}$. This function $\left(\phi_{2}\right)$ can be different for different values of $\xi_{1}$ and, hence, our notation shows an explicit dependence of $\phi_{2}$ on $\xi_{1}$. The search for optimal $\phi_{1}$ and $\phi_{2}$ is performed using the same elastic energy function and the computational implementation is based on multiple uses of the dynamic programming algorithm.

3. Optimal Deformation: The process of matching points and curves across faces also results in the optimal elastic deformation between faces. As a basic unit for this deformation, we obtain geodesic paths between corresponding (matched) curves from one face to the other; these paths provide optimal elastic deformations between those curves. Each face is represented as an indexed collection of curves and, thus, a composition of these individual geodesics provides a global geodesic (and an optimal elastic deformation) between the two faces. A major advantage of a Riemannian framework is that it provides geodesic paths, 
between faces, that are necessary for a full statistical analysis. This is often not feasible in case of global distance based matching algorithms, e.g. in case of Gromov-Hausdorff distance [3].

The main differences between this paper and our earlier papers are as follows:

1. In Samir et al. [35] and [34], we used the level curves of the height function to define facial curves. Since these curves are planar, we used shape analysis of planar curves [23] to compare and deform faces; nonlinear matching problem was not studied here (that is, the mapping was fixed to be linear). The metric used was a non-elastic metric and the shooting method was used for computing geodesics between planar curves. Consequently, this representation is not fully invariant to rigid motions of the face.

2. In Samir et al. [36], we used the level curves of the geodesic distance function that resulted in 3D curves. We used a non-elastic metric and a path-straightening method to compute geodesics between these curves [22]. Here also, the matching was not studied and the correspondence of curves and points across faces was simply linear.

3. In the current paper, we extend the earlier Riemannian framework to be fully elastic by defining a formal coordinate system on facial surfaces and using that to solve the matching, deformation, and comparison problems. Two types of elastic matchings are developed - one for matching the curves and one for matching points on the curves. Curves are represented using a square-root velocity function that has recently been introduced for studying shapes of elastic curves $[18,17]$.

The rest of this paper is organized as follows. We introduce the Darcyan coordinate system $\left(\xi_{1}, \xi_{2}\right)$ on facial surfaces in Section 2. In Section 3, we summarize ideas from [18] on elastic matching of points on closed curves in $\mathbb{R}^{3}$ and the resulting deformations of level curves of $\xi_{1}$, while in Section 4 , we use ideas from dynamic programming to match curves on facial surfaces and to deform facial surfaces. In Section 5 , we present some examples on elastic deformation facial surfaces and some their use for applications in face classification. We finish the paper with a summary in Section 6 .

\section{Curvilinear Coordinate System}

The starting point of our framework is to define an intrinsic coordinate system on each facial surface that is natural for studying shape variability. This system is used to decompose elastic matching/deformation of facial surfaces into simpler, more elementary components. In this system the level curves are neither straight lines nor circles; these are curves that lie completely on the surface and locally match the geometry of the surface. Let $S$ be a facial surface assumed to be a two-dimensional differentiable manifold that contains its boundaries. This is obtained in practice by filling the holes in the data obtained from 3D scanners, and by smoothly interpolating the resulting mesh to proper dimensionality of all patches on the surface. Furthermore, $S$ is endowed with a Riemannian structure using the Euclidean metric in $\mathbb{R}^{2}$, the tangent space at any interior point of $S$. Let $r \in S$ denote the tip of the nose on $S$.

Proposition 1 For any point p, there exists a shortest geodesic, connecting $r$ and $p$, that lies completely in S. The shortest geodesic may not be unique, but there exists at least one.

The proof comes from the fact that $S$ is a finite-dimensional, complete, and differentiable manifold. Based on this proposition, one can define a smooth function on $S$ using geodesic distances as follows:

Definition 1 For any point $p \in S$, define a function $f: S \mapsto \mathbb{R}$ such that $f(p)$ is the length of the shortest geodesic from $r$ to $p$.

Since there exists a shortest geodesic from $r$ to $p$, for all $p$, the function $f$ is well defined. Also, since $S$ is a differentiable manifold, the function $f$ is a continuous function.

Remark: We caution the reader that this use of geodesic on facial surfaces is different from the later construction of geodesic paths between two curves or two surfaces under appropriate Riemannian metrics. Here, we compute geodesics on $S$, a two-dimensional manifold with boundaries, to define $f$, while later on we will compute geodesics on infinite-dimensional spaces of curves and surfaces to realize optimal deformations between their elements. 

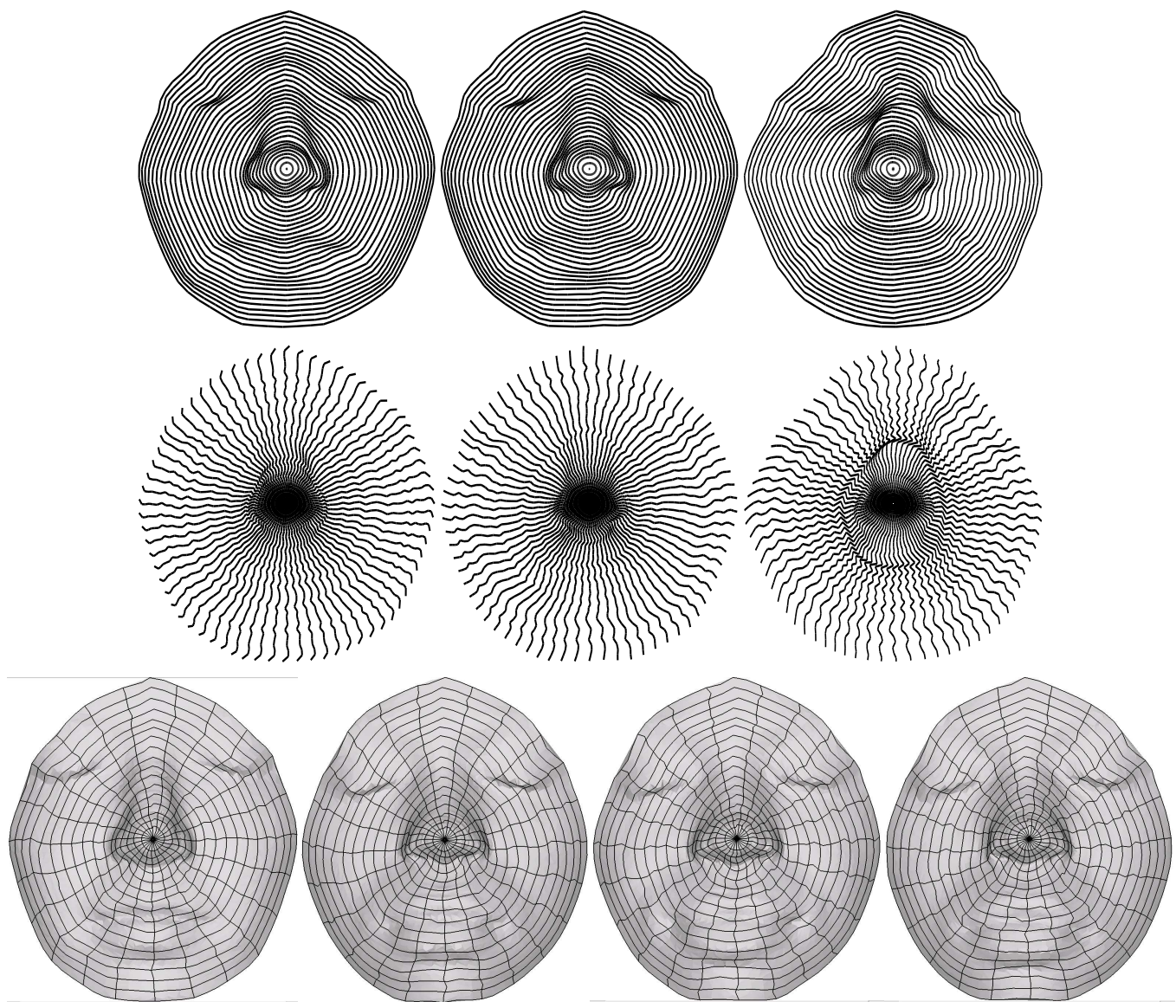

Fig. 1 Top row: Level curves of $\xi_{1}$ and middle row: level curves of $\xi_{2}$ for three different facial surfaces. Bottom row: full Darcyan coordinate system on some faces.

Define a coordinate system on $S$ using $f$ as follows. Define the level curves of $f$ as the level curves of the first coordinate $\xi_{1}$, the value of $\xi_{1}$ being equal to the value of $f$. Shown in the top row of Figure 1 are examples of these curves for three different faces. Next, use the streamlines of $\nabla f$, starting from $r$, as the level curves of the second coordinate $\xi_{2}$. The value of $\xi_{2}$ along such curves will be dependent on the initial directions of these streamlines. Shown in the second row of Figure 1 are examples of these curves. Together, these two coordinates provide a well-defined, orthogonal coordinate system.

Lemma 1 For a given smooth surface $S$, the Darcyan coordinate system is well defined for all of $S$. It has the property that the level curves of the two coordinates intersect at right angles.

Following Grenander et al. [13], we will call it the Darcyan coordinate system, named after the famous shape analyst D'Arcy Thompson.

Note that one can also develop this system by forming a polar coordinate system on the tangent space, $T_{r}(S) \equiv \mathbb{R}^{2}$, and by projecting level curves (circles and radial lines) onto $S$ using the exponential maps (or geodesics along $S$ ) [5]. For this reason, these level curves of $\xi_{1}$ have also being called geodesic (polar) circles. Bronstein et al. [4] used largest geodesic circles to prune facial surfaces using multiple reference points; in that context, these circles were called geodesic masks. Some authors, e.g. [32], have used this system for matching features across faces but we are interested in a full shape analysis, that is, matching, deformation and comparisons of faces. 


\section{Elastic Matching/Deformation of Darcyan Level Curves}

Once the Darcyan coordinate system is established, the task of elastically matching two surfaces is that of finding a mapping $\phi: S_{1} \mapsto S_{2}$ that is optimal under the elastic metric. In our approach, $\phi$ can be decomposed into simpler components. There are two ways of decomposing $\phi\left(\xi_{1}, \xi_{2}\right)$ : (i) into $\phi_{1}\left(\xi_{1}\right) \phi_{2}\left(\xi_{2} ; \xi_{1}\right)$ and (ii) into $\phi_{2}\left(\xi_{2}\right) \phi_{1}\left(\xi_{1} ; \xi_{2}\right)$. In the former decomposition, we first match the level curves of $\xi_{1}$, to find $\phi_{1}$, and then for each pair of matched curves we find $\phi_{2}$. This procedure is reversed for the latter decomposition. Similar to [36], we choose the former in this paper since the level curves of $\xi_{1}$ are more interesting and visually more appealing for shape analysis. We remark that the level curves of $\xi_{2}$ form facial profiles, similar to the idea used in [2] for face profiling.

We start by describing the estimation of $\phi_{2}$. Let $C_{\xi_{1}}^{1}$ and $C_{\xi_{1}^{\prime}}^{2}$ be two arbitrary level curves of the first coordinate in facial surfaces $S_{1}$ and $S_{2}$, respectively. Our goal is to find a mapping $\phi_{2}: \xi_{2} \mapsto \xi_{2}^{\prime}$ that is a diffeomorphism and it minimizes the elastic distance (to be defined) between the two curves. We treat the two curves as closed, parameterized curves in $\mathbb{R}^{3}$ with fixed origins for parameterizations. Furthermore, we rescale them to be of the same length, say $2 \pi$. This viewpoint allows us to use one of many methods already available for an elastic analysis of closed curves. The key idea in elastic analysis is that the mapping $\phi_{2}$ is nonlinear, i.e. points that are matched together are at unequal distances from their origins. Such a matching can be considered as an elastic matching, as one curve has to (locally) stretch, compress and bend to match the other. Several authors, starting with Younes [44], followed by Michor \& Mumford [29], Mio et al. [31], and others have studied it for planar shapes. More recently Joshi et al. $[18,17]$ have extended it to curves in $\mathbb{R}^{n}$ using an efficient representation of curves. Several authors, including Yezzi \& Mennucci [43], have also used Riemannian metrics on spaces of curves but mainly to study evolution of curves rather than for shape analysis. Here we adopt the approach presented in Joshi et al. [18,17] because it greatly simplifies the elastic shape analysis. The main steps are: (i) define a space of closed curves of interest, (ii) impose a Riemannian structure on it using the elastic metric, and (iii) compute geodesic paths under this metric. These geodesic paths can then be interpreted as optimal elastic deformations of curves.

For the interval $I \equiv[0,2 \pi]$, let $\beta: I \rightarrow \mathbb{R}^{3}$ be a parameterized curve with a non-vanishing derivative everywhere. We represent its shape by the function $q: I \rightarrow \mathbb{R}^{3}$ defined as: $q(s)=\frac{\dot{\beta}(s)}{\sqrt{\|\dot{\beta}(s)\|}} \in \mathbb{R}^{3}$. Here, $\|\cdot\|$ is the standard Euclidean norm in $\mathbb{R}^{3}$. The quantity $\|q(s)\|$ is the square-root of the instantaneous speed on the curve $\beta$, whereas the ratio $\frac{q(s)}{\|q(s)\|}$ is the instantaneous direction at the point $s \in[0,2 \pi)$ along the curve. Let $\mathcal{Q} \equiv\left\{q=\left(q_{1}, q_{2}, q_{3}\right) \mid q(s): I \rightarrow \mathbb{R}^{3},\|q(s)\| \neq 0, \forall s\right\}$ be the space of all square integrable functions in $\mathbb{R}^{3}$. The closure condition for a curve $\beta$ requires that $\int_{I} \dot{\beta}(s) d s=0$, which translates to $\int_{I}\|q(s)\| q(s) d s=0$. We define a mapping $\mathcal{G}: \mathcal{Q} \mapsto \mathbb{R}^{4}$, with components:

$$
\mathcal{G}_{1}=\int_{I} q_{1}(s)\|q(s)\| d s, \mathcal{G}_{2}=\int_{I} q_{2}(s)\|q(s)\| d s, \mathcal{G}_{3}=\int_{I} q_{3}(s)\|q(s)\| d s, \mathcal{G}_{4}=\int_{I}\|q(s)\|^{2} d s .
$$

The space obtained by the inverse image $\mathcal{C}=\mathcal{G}^{-1}(0,0,0,2 \pi)$ is the space of all closed curves of length $2 \pi$, and this representation is invariant to translation and scaling. $\mathcal{C}$ is endowed with a Riemannian structure using the metric: for any two tangent vectors $v_{1}, v_{2} \in T_{q}(\mathcal{C})$, we define

$$
\left\langle v_{1}, v_{2}\right\rangle=\int_{I} v_{1}(s)^{T} v_{2}(s) d s
$$

It is shown in [18] that this metric is equivalent to the elastic metric described in [31].

Next, we want a tool to compute geodesic paths between arbitrary elements of $\mathcal{C}$. There have been two prominent numerical approaches for computing geodesic paths on nonlinear manifolds. One approach uses the shooting method $([23,31])$ where, given a pair of shapes, one finds a tangent direction at the first shape such that its image under the exponential map reaches as close to the second shape as possible. We will use another, more stable approach that uses path-straightening flows to find a geodesic between two shapes. In this approach, the given pair of shapes is connected by an initial arbitrary path that is iteratively "straightened" so as to minimize its length. The path-straightening method, proposed by Klassen et al [22], overcomes some of the practical limitations in the shooting method. Other authors, including Schmidt et al. [37] and Glaunes et al [10], have also presented other variational techniques for finding optimal matches. 

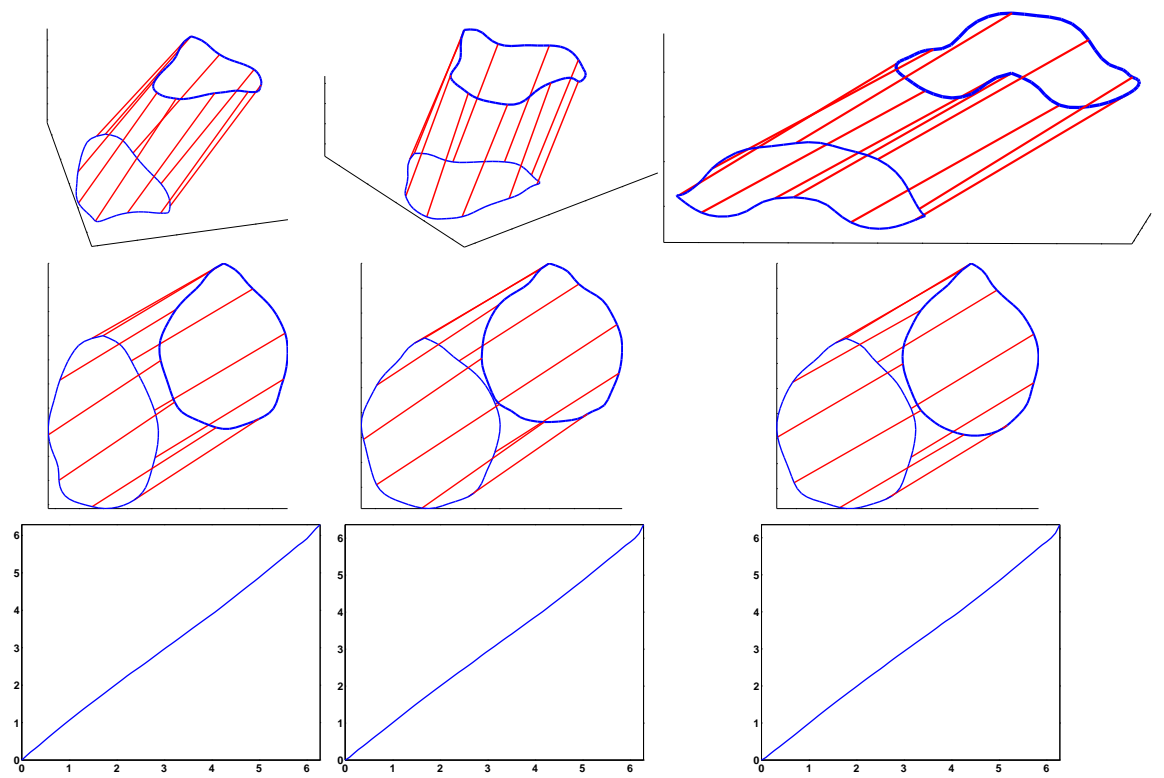

Fig. 2 Elastic matching of facial curves. The top and the middle rows show two different views of the matching points across the curves, while the bottom panels show optimal matching functions $\phi_{2}^{*}$.

Given two curves, represented by $q^{0}$ and $q^{1}$, our goal is to find a geodesic between them in $\mathcal{C}$. Let $\alpha:[0,1] \rightarrow \mathcal{C}$ be any path connecting $q^{0}, q^{1}$ in $\mathcal{C}$, i.e. $\alpha(0)=q^{0}$ and $\alpha(1)=q^{1}$. Then, the critical points of the energy

$$
E[\alpha]=\frac{1}{2} \int_{0}^{1}\langle\dot{\alpha}(t), \dot{\alpha}(t)\rangle d t,
$$

with the inner product defined in Eqn. 1, are geodesics in $\mathcal{C}$ (this result is true on a general manifold [39]). As described by Klassen et al. [22] (for general shape manifolds), and Joshi et al. [18] (for this particular representation of curves), one can use a gradient approach to find a critical point of $E$ and reach a geodesic. The distance between the two curves $q^{0}$ and $q^{1}$ is given by the length of the geodesic $\alpha$ :

$$
d_{c}\left(q^{0}, q^{1}\right)=\int_{0}^{1}\left(\left\langle\alpha^{\prime}(t), \alpha^{\prime}(t)\right\rangle\right)^{1 / 2} d t
$$

We will call this the elastic distance between the curves represented by $q^{0}$ and $q^{1}$.

Similar to Kendall's shape formulation [20], we define shapes of curves as invariant under rigid motions, uniform scaling and other such "shape-preserving" transformations. Since translation, scaling, and location of origin are already accounted for, we need to remove the variability associated with rotations and originpreserving (positive) diffeomorphisms on $I$. In other words, the shape space for analyzing shapes of closed curves in $\mathbb{R}^{3}$ is given by $\mathcal{S}=\mathcal{C} /(S O(3) \times \mathcal{D})$, where $S O(3)$ is the three-dimensional rotation group and $\mathcal{D}$ is the set of origin-preserving positive diffeomorphisms on $I$. The task of finding a geodesic in $\mathcal{S}$ is that of finding a geodesic in $\mathcal{C}$ that achieves the distance:

$$
d_{s}\left(q_{1}, q_{2}\right)=\min _{\gamma \in \mathcal{D}, O \in S O(3)} d_{c}\left(q_{1}, \gamma \cdot\left(O q_{2}\right)\right),
$$

where $\gamma \cdot q_{2}$ is the re-parametrization of $q_{2}$ according to the formula: $\sqrt{\dot{\gamma}} q_{2}(\gamma)$. As described in [17], one can solve for this minimization on $\mathcal{D} \times S O(3)$ using a gradient approach; please refer to that paper for details. We will illustrate these ideas using some examples. Some examples of elastic matching of corresponding points across facial curves is shown in Figure 2; it shows three examples of matching pairs of facial curves and the corresponding optimal matching functions $\phi_{2}^{*}$. The optimal $\gamma$, that minimizes the right side of Eqn. 3 , provides the component $\phi_{2}^{*}$ of the optimal mapping $\phi^{*}$. Figure 3 illustrates the idea of optimal elastic deformations between facial curves. It shows the geodesic paths between facial curves in $\mathcal{S}$ under the elastic 


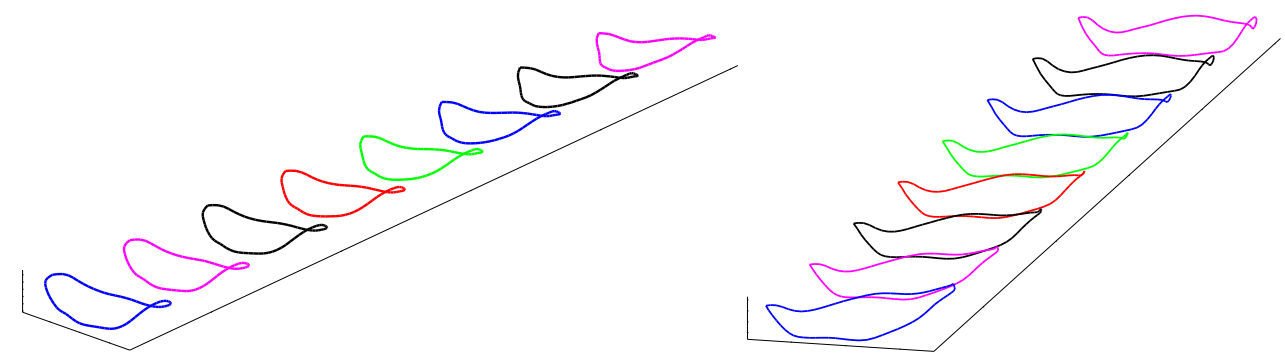

Fig. 3 Examples of optimal elastic deformations between facial curves extracted as level curves of $\xi_{1}$.

metric. In each case, the first and the last curve are the examples of facial curves and the intermediate curves are points along a geodesic path connecting them in $\mathcal{S}$.

\section{Elastic Matching of Curves Across Facial Surfaces}

Now that we have tools for elastically deforming arbitrary facial curves into each other, we consider the problem of matching curves across two faces. Each facial surface is denoted by an indexed collection of facial curves, $S_{i} \equiv\left\{C_{\xi_{1}}^{i} \mid \xi_{1} \in[0, L]\right\}, i=1,2$. (We remind the reader that $C_{\xi_{1}}^{i}$ is the level curve of first Darcyan coordinate at the value $\xi_{1}$ for the facial surface $S_{i}, i=1,2$.) Our goal is to find $\phi_{1}$ that optimally matches curves across these collections. We initially formulate the problem in the continuum, but will later discretize the domain to reach a computational solution. Let $\phi_{1}:[0, L] \mapsto[0, L]$ be a diffeomorphic mapping that minimizes the cost function:

$$
E_{s}\left[\phi_{1} ; 0, L\right]=\int_{0}^{L} d_{s}\left(C_{\xi_{1}}^{1}, C_{\phi_{1}\left(\xi_{1}\right)}^{2}\right)^{2} d \xi_{1},
$$

where $d_{s}$ is as described in Eqn. 3. The solution requires a search over the infinite-dimensional space of all diffeomorphisms from $[0, L]$ to itself. There exists a computational approach to solving this type of problem in situations where the domain and the range of $\phi_{1}$ are sampled evenly using a finite number of points. Termed as dynamic time warping, this technique has been used for matching points on curves by several authors, include Sebastian et al. [38] and Mio et al. [31]. For notational convenience we will rescale $\xi_{1}$ so that it takes values in $[0,1]$, and we seek an optimal $\phi_{1}$ on $\mathcal{D}_{[0,1]}$, the set of all diffeomorphisms from $[0,1]$ to itself.

Let each facial surface be sampled evenly in $\xi_{1}$ at $n$ points to give an indexed collection of $n$ curves, each an element in $\mathcal{C}$. This results in an $n \times n$ graph, of the type shown in Figure 4 , whose nodes denote possible matchings of corresponding curves from the two surfaces. The goal is now to find a piecewise linear $\phi_{1} \in \mathcal{D}_{[0,1]}$, i.e. a curve that passes through the nodes on the graph, that minimizes the energy function given in Eqn. 4, and we do so using dynamic programming (DP). DP is useful in finding optimal paths between any two points on a finite graph when the cost associated with the path is additive over its segments. The piecewise-linear path between the points $(0,0)$ to $(1,1)$ is constrained to be such that: (i) the slope is always positive along the path, and (ii) it minimizes the elastic energy. To evaluate the elastic energy we will need the term $d_{s}\left(C_{(i / n)}^{1}, C_{(j / n)}^{2}\right)$ for all $i, j$, as described in the previous section.

Consider a uniform, $n \times n$ grid in $\mathbb{R}^{2}$ such that the bottom left maps to $(0,0)$ and the top right maps to indices $(1,1)$ on this grid, as shown in Figure 4 . For any point $(i / n, j / n)$, for $0<i, j \leq n$, let $N_{i j}$ be the set of indices that are allowed to go to $(i / n, j / n)$. In principle, all the points $(k / n, l / n)$ such that $k<i$ and $l<j$ are valid, but one may restrict to a smaller set to seek a faster approximation. Let $L(k, l ; i, j)$ denote a straight line joining the pairs $(k / n, l / n)$ and $(i / n, j / n)$. Then, the iterative optimization problem is:

$$
(\hat{k}, \hat{l})=\underset{(k, l) \in N_{i j}}{\operatorname{argmin}} E_{s}[L(k, l ; i, j) ; k / n, i / n],
$$

with $E_{s}$ as defined in Eqn. 4. Define the minimum energy of reaching the point $(i / n, j / n)$, in an iterative fashion as:

$$
H_{s}(i / n, j / n)=E_{s}[L(\hat{k}, \hat{l} ; i, j), \hat{k} / n, i / n]+H_{s}(\hat{k} / n, \hat{l} / n), \quad \text { with } H(0,0)=0 .
$$



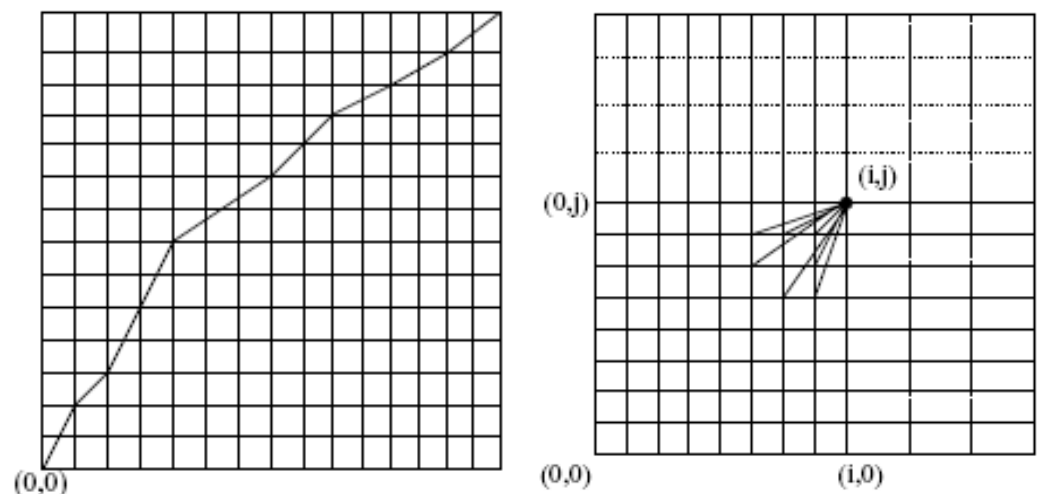

Fig. 4 Left: An example of a $\phi_{1}$ function restricted to a finite graph. Right: An illustration of some nodes that are allowed to go to $(i / n, j / n)$ point on the graph.
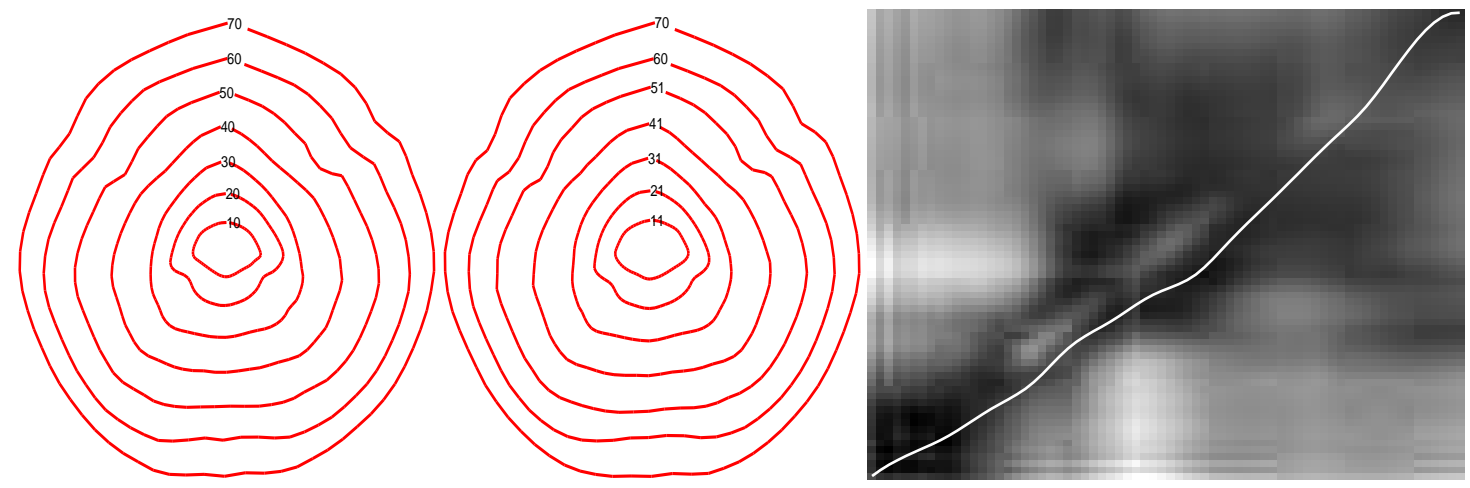

Fig. 5 The left two panels show a sub-sampled set of matched curves- 10 with 11,20 with 21, etc - across the two collections representing $S_{1}$ and $S_{2}$. The rightmost panel shows the optimal matching function $\phi_{1}^{*}$ between the two collections.

The optimization problem in Eqn. 5 is solved sequentially for each point $(i / n, j / n)$, starting from $(0,0)$ and increasing $i / n, j / n$ until the last point $(1,1)$. Tracing the path that results in the optimal elastic energy $H_{s}(1,1)$ provides a discrete version of the optimal $\phi_{1}$. The set of nodes on the graph, contained in optimal $\phi_{1}$, provide the matching pairs in the two collections. Shown in Figure 5 is an example of this idea for $n=70$. The rightmost panel shows a pictorial view of the function $H$ with darker shade corresponding to lower energy. Overlaid on this picture is the plot of optimal $\phi_{1}$ for this case. Shown in the left two panels are the corresponding pairs of curves in the two faces. Only the matching curves, associated with the nodes that lie on optimal $\phi_{1}$, are shown for clarity. According to this figure, the $10^{\text {th }}$ curve in the first surface matches the $11^{\text {th }}$ curve in the second, the $50^{\text {th }}$ curve in the first surface matches the $51^{\text {st }}$ curve in the second, and so on.

\section{Elastic Deformation of Facial Surfaces}

The previous two sections provide the solutions for solving optimal mappings $\phi_{2}^{*}$ (Section 3) and $\phi_{1}^{*}$ (Section 4). Together they provide an elastic matching of points across the two facial surfaces according to $\phi^{*}\left(\xi_{1}, \xi_{2}\right)=$ $\phi_{1}^{*}\left(\xi_{1}\right) \phi_{2}^{*}\left(\xi_{2} ; \xi_{1}\right)$.

The construction of geodesic paths between Darcyan curves in Section 3, using an elastic metric, allows us to realize optimal deformations between surfaces. Applying this idea repeatedly to each pair of registered curves gives us a full elastic deformation from one face to another. If we view each facial surface as an indexed collection of curves, indexed by $\xi_{1}$, this idea provides a path on the space of such collections, in going from one face to another. We will denote this path by $\Omega$, where $\Omega(t)$, for any $t \in[0,1]$ is an indexed collection of 


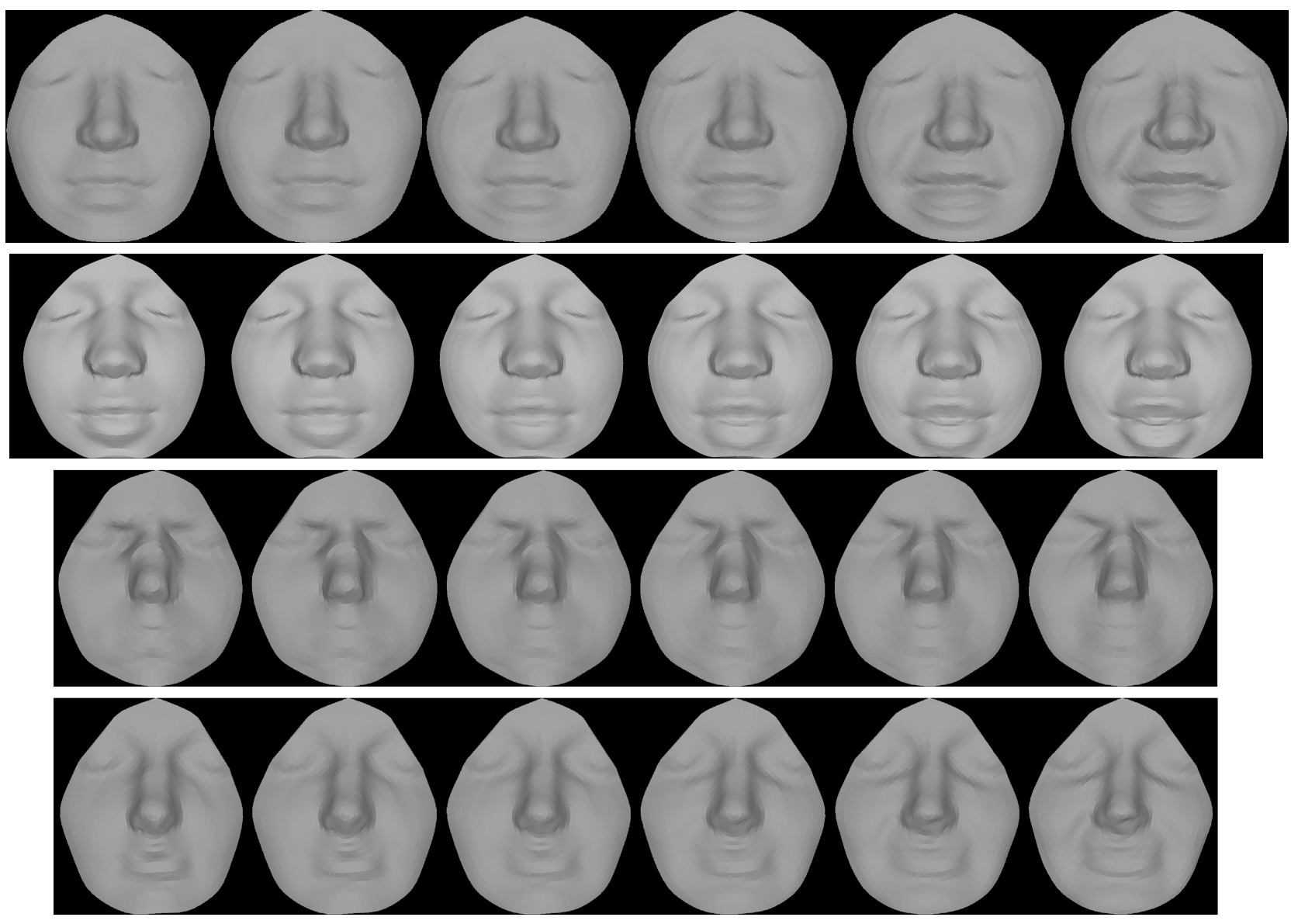

Fig. 6 Optimal deformations of facial surfaces into each other, both belonging to the same person, under the elastic energy.

points in $\mathcal{S}$. This path is optimal in the sense that it minimizes the total length:

$$
d\left(S_{1}, S_{2}\right) \equiv \int_{0}^{1} d_{s}\left(C_{\xi_{1}}^{1}, C_{\phi_{1}^{*}\left(\xi_{1}\right)}^{2}\right) d \xi_{1}
$$

Shown in Figures 6 - 7 are examples of these deformations. Figure 6 shows four examples of deforming facial surfaces, one into another, when both the faces belong to the same person. Similarly, Figure 7 shows four examples of elastic deformations when the faces come from different persons.

Shown in Figure 8 is an example of comparison of deformations obtained under elastic and non-elastic metrics. The top row shows an example of non-elastic, or bending-only, deformation as described in Samir et al. [36], while the bottom row shows the elastic deformation introduced in this paper. Although the differences between the two deformations are not apparent visually, a closer inspection reveals a smoother deformation, especially around the lips, in the elastic case.

This solution also provides an elastic distance (Eqn. 6) between individual facial surfaces. To illustrate the usefulness of this distance, we show a matrix of pairwise distances between 12 faces in Figure 9, six of which belong to the same person while the remaining six belong to different persons. In this display, the distances have been scaled to take value between 0 and 1 . As expected, the distances between faces of same person are much smaller compared to the distances between faces of different persons.

Since the Darcyan system relies critically on the location of the reference point (tip of the nose), and there is a possibility of numerical error in estimating this point, how stable is the framework presented here to such numerical errors? We have studied this issue experimentally and some results are presented in Figure 10. The left two panels show the level curves of $\xi_{1}$ on the same facial surface when the reference point is 

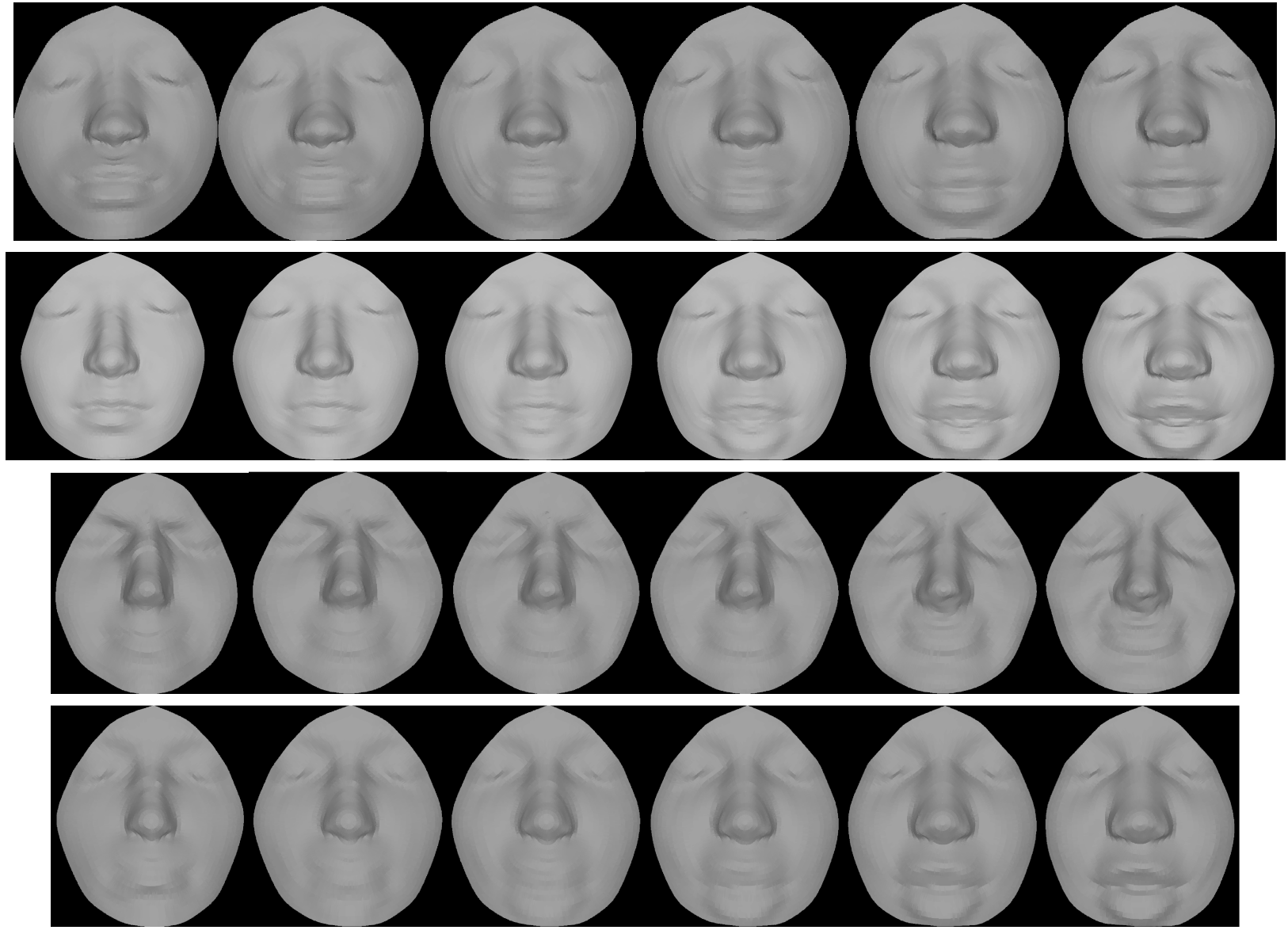

Fig. 7 Optimal deformations of a facial surface into another, belonging to a different person, under the elastic energy.

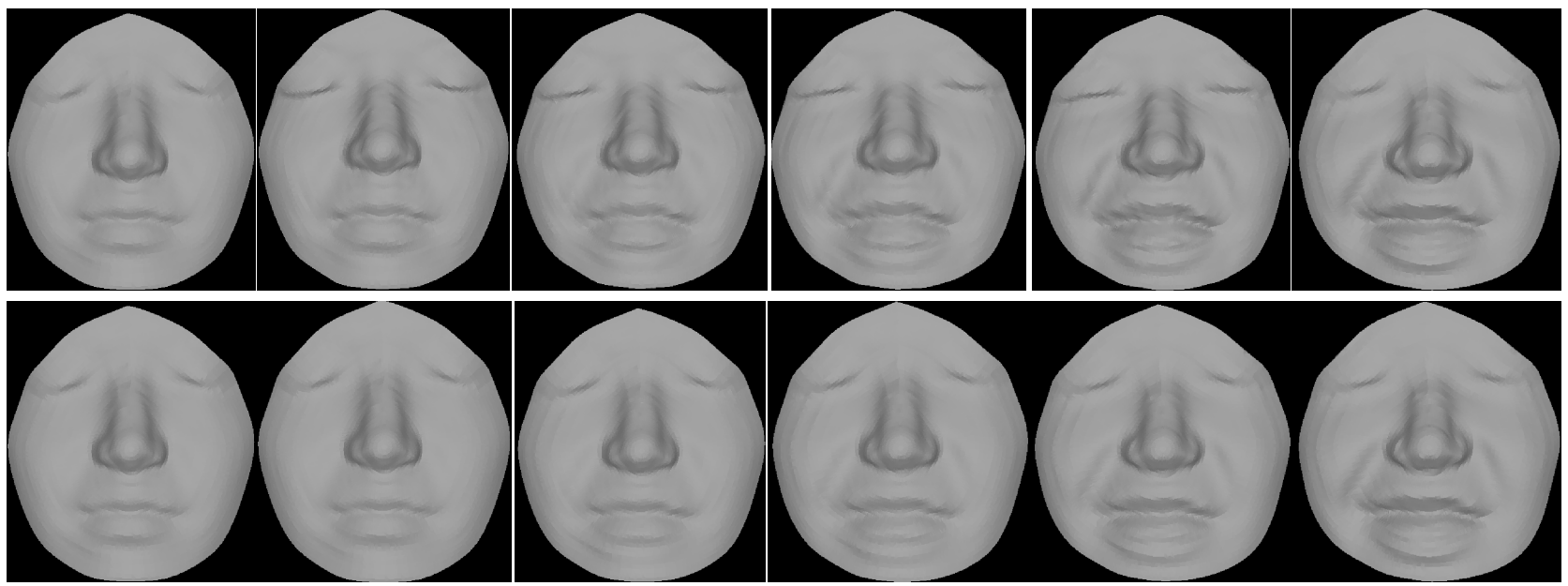

Fig. 8 Elastic versus non-elastic deformation. For the same pair of faces we show geodesic paths (deformations) using the non-elastic metric (top, as described in Samir et al. [36]) and the elastic metric (bottom) used in this paper. 


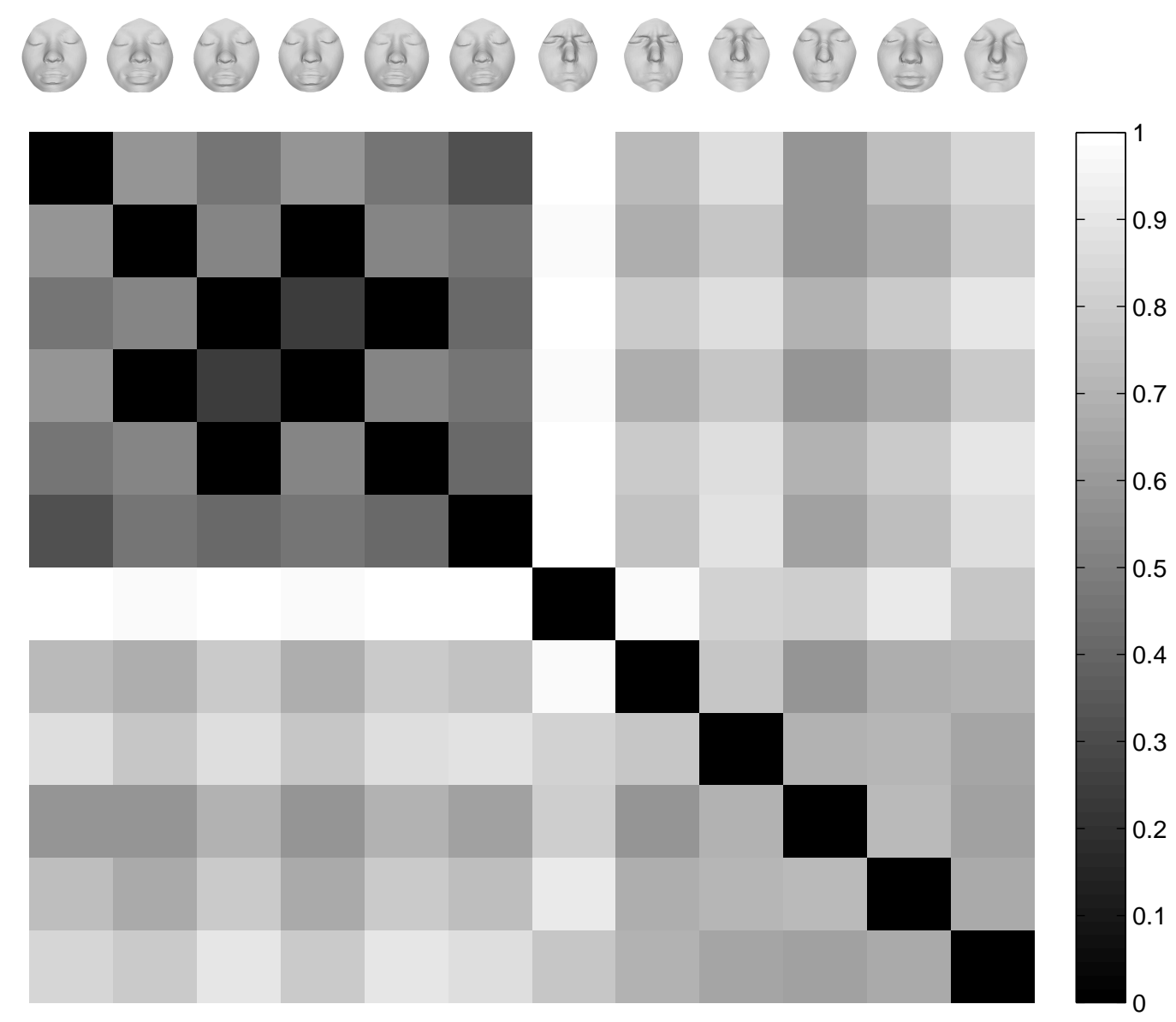

Fig. 9 Matrix of (scaled) pairwise distances between 12 facial surfaces. The first six faces belong to the same person while the remaining six are all different individuals.
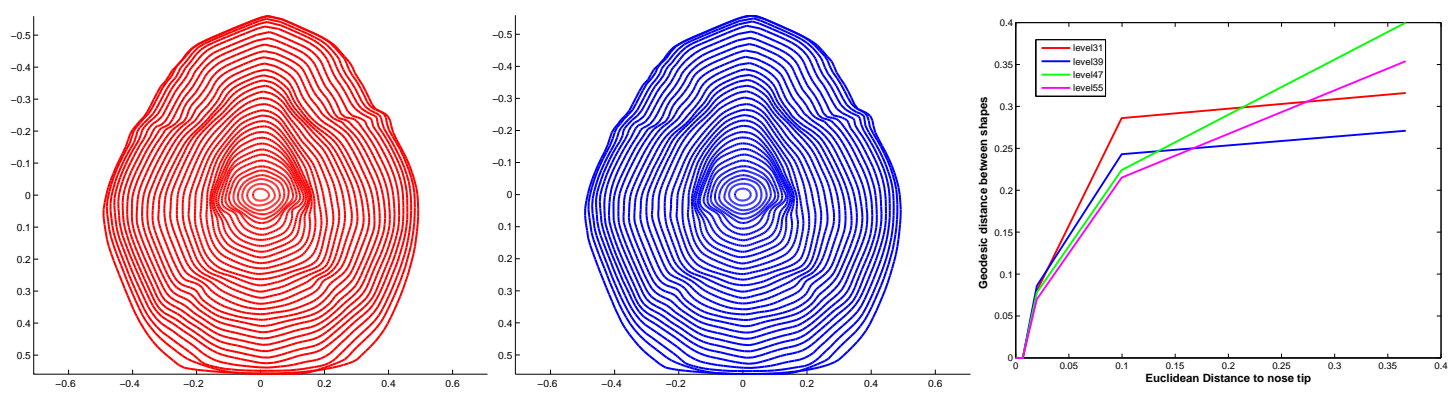

Fig. 10 Left two panels show level curves of Darcyan coordinate $\xi_{1}$ when the location of reference point is changed slightly. The panels shows the variation in shapes of level curves versus changes in locations of reference points, for curves at four levels of $\xi_{1}$.

located in a slightly different spot. The right plot shows the variation in shapes of levels curves, at four different levels, versus changes in the location of the reference point. For example, one curve in this plot shows the change in shapes of level curve 31, measured using the geodesic distance $d_{s}$, when the reference point is moved away from the actual tip of the nose. These variations are found to be sub-linear, implying a stability of shape analysis with respect to reference point location. 


\section{Summary}

We have presented a Riemannian framework for analyzing shapes of facial surfaces for the purposes of matching, comparing, and deforming them. This approach uses a Darcyan coordinate system, a system that is intrinsic to a facial surface. This system is centered at the tip of the nose and its two coordinates $\xi_{1}$ and $\xi_{2}$ specify distances from this tip and the plane of symmetry, respectively. In this coordinate system, the task of elastic matching and deforming is broken into two distinct components. One component matches level curves of each surfaces, and the other component matches points along each pair of matched curves. These tasks are performed using geodesics on appropriate manifolds of curves and surfaces, with Riemannian metrics that measure elastic deformation. In other words, geodesics provide the optimal deformations of curves and surfaces across faces.

\section{Acknowledgements}

The authors would like to thank CNRS, France, for a visiting Professorship to Anuj Srivastava, and Groupe des Ecole de Telecommunications for its support. This research was also supported in part by the grants ARO W911NF-04-1-0268, ARO W911NF-04-1-0113, and AFOSR FA9550-06-10324. We also thank the two reviewers and the associate editor for their feedback in improving this paper.

\section{References}

1. P.J. Besl and N.D. McKay. A method for registration of 3-D shapes. IEEE Transactions on Pattern Analysis and Machine Intelligence, 14(2):239-256, February 1992.

2. C. Beumier and M. Acheroy. Automatic 3D face authentication. Journal of Image and Vision Computing, $18(4): 315-321,2000$.

3. A. M. Bronstein, M. M. Bronstein, and R. Kimmel. Efficient computation of isometry-invariant distances between surfaces. SIAM Journal on Scientific Computing, 28(5):1812-1836, 2006.

4. A. M. Bronstein, M.M. Bronstein, and R. Kimmel. Three-dimensional face recognition. International Journal of Computer Vision, 64(1):5-30, 2005.

5. M. P. Do Carmo. Differential Geometry of Curves and Surfaces. Prentice Hall, Inc., 1976.

6. K. I. Chang, K. W. Bowyer, and P. J. Flynn. Multiple nose region matching for 3D face recognition under varying facial expression. IEEE Transactions on Pattern Analysis and Machine Intelligence, 28(10):1695-1700, 2006.

7. G. Charpiat, O. Faugeras, and R. Keriven. Approximations of shape metrics and application to shape warping and empirical shape statistics. Journal of Foundations of Computational Mathematics, 5(1):1-58, 2005.

8. H. Chui and A. Rangarajan. A new algorithm for non-rigid point matching. In Proc. of IEEE Conferences on CVPR, pages $44-51,2000$.

9. J. Glaunes, A. Trouvé, and L. Younes. Diffeomorphic matching of distributions: A new approach for unlabelled point-sets and sub-manifolds matching. In CVPR (2), pages 712-718, 2004.

10. J. Glaunes, A. Trouve, and L. Younes. Modeling planar shape variation via hamiltonian flows of curves. In H. Krim and A. Yezzi, editors, Statistics and Analysis of Shapes. Birkhauser, 2006.

11. U. Grenander. General Pattern Theory. Oxford University Press, 1993.

12. U. Grenander and M. I. Miller. Computational anatomy: An emerging discipline. Quarterly of Applied Mathematics, LVI(4):617-694, 1998.

13. U. Grenander, A. Srivastava, and S. Saini. A pattern-theoretic characterization of biological growth. IEEE Transactions on Medical Imaging, 26(5):648-659, 2007.

14. S. Gupta, M. K. Markey, J. Aggarwal, and A. C. Bovik. Three dimensional face recognition based on geodesic and euclidean distances. In Proceedings of ISET/SPIE Symposium on Electronic Imaging: Vision Geometry XV, 2007.

15. P. W. Hallinan, G. G. Gordon, A. L. Yuille, P. Giblin, and D. Mumford. Two- and Three-Dimensional Patterns of Face. A. K. Peters, 1999.

16. C. Hesher, A. Srivastava, and G. Erlebacher. A novel technique for recognizing faces using range images. In Proceedings of ISSPA, 2003, Paris, France, 2003.

17. S. Joshi, A. Srivastava, E. Klassen, and I. H. Jermyn. Removing shape-preserving transformations in squareroot elastic (SRE) framework for shape analysis of curves. In Proc. of Sixth Workshop on Energy Minimization Methods in CVPR (EMMCVPR), 2007. 
18. S. H. Joshi, E. Klassen, A. Srivastava, and I. H. Jermyn. An efficient representation for computing geodesics between n-dimensional elastic shapes. In IEEE Conference on Computer Vision and Pattern Recognition (CVPR), June 2007.

19. I. A. Kakadiaris, G. Passalis, G. Toderici, M. N. Murtuza, N. Karampatziakis, and T. Theoharis. Threedimensional face recognition in the presence of facial expressions: An annotated deformable model approach. IEEE Transactions on Pattern Analysis and Machine Intelligence, 29(4):1-10, 2007.

20. David G. Kendall. Shape manifolds, procrustean metrics and complex projective spaces. Bulletin of London Mathematical Society, 16:81-121, 1984.

21. M. Kilian, N. J. Mitra, and H. Pottmann. Geometric modeling in shape space. In Proceedings of SIGGRAPH, 2007.

22. E. Klassen and A. Srivastava. Geodesics between 3D closed curves using path straightening. In European Conference on Computer Vision, vol. I, pages 95-106, 2006.

23. E. Klassen, A. Srivastava, W. Mio, and S. H. Joshi. Analysis of planar shapes using geodesic paths on shape spaces. IEEE Trans. Pattern Analysis and Machine Intelligence, 26(3):372-383, 2004.

24. C. Kotropoulos, A. Tefas, and I. Pitas. Frontal face authentication using morphological elastic graph matching. IEEE Transactions on Image Processing, 9(4):555-560, April 2000.

25. N. Litke, M. Droske, M. Rumpf, and P. Schröder. An image processing approach to surface matching. In M. Desbrun and H. Pottmann, editors, Third Eurographics Symposium on Geometry Processing, pages 207-216, 2005.

26. X. Lu and A. K. Jain. Deformation analysis for 3D face matching. In Proc. 7th IEEE Workshop on Applications of Computer Vision, pages 99-104. Breckenridge, CO, 2005.

27. X. Lu, A. K. Jain, and D. Colbry. Matching 2.5D face scans to 3D models. IEEE Transactions on Pattern Analysis and Machine Intelligence, 28(1):31-43, Jan. 2006.

28. F. Memoli and Sapiro G. A theoretical and computational framework for isometry invariant recognition of point cloud data. Foundations and Computational Mathematics, 5(3):313-347, 2005.

29. P. W. Michor and D. Mumford. Riemannian geometries on spaces of plane curves. J. Eur. Math. Soc., 8:1-48, 2006.

30. M. I. Miller and L. Younes. Group actions, homeomorphisms, and matching: A general framework. Intl. Journal of Computer Vision, 41(1/2):61-84, 2001.

31. W. Mio, A. Srivastava, and S. H. Joshi. On shape of plane elastic curves. International Journal of Computer Vision, 73(3):307-324, 2007.

32. I. Mpiperis, S. Malassiotis, and M. G. Strintzis. 3-D face recognition with the geodesic polar representation. IEEE Transactions on Information Forensics and Security, 2(3):537 - 547, 2007.

33. R. Osada, T. Funkhouser, B. Chazells, and D. Dobkin. Matching 3D models with shape distributions. In IEEE Shape Modeling International, May 2001.

34. C. Samir, A. Srivastava, and M. Daoudi. Three-dimensional face recognition using shapes of facial curves. IEEE Transactions on Pattern Analysis and Machine Intelligence, 28(11):1858-1863, 2006.

35. C. Samir, A. Srivastava, and M. Daoudi. 3D face recognition using shapes of facial curves. In Proceedings of ICASSP, 2006, Special Session on Statistical inferences on nonlinear manifolds with applications in signal and image processing, May, 2006.

36. C. Samir, A. Srivastava, M. Daoudi, and E. Klassen. An intrinsic framework for analysis of facial surfaces. International Journal of Computer Vision, in review, 2007.

37. F. R. Schmidt, M. Clausen, and D. Cremers. Shape matching by variational computation of geodesics on a manifold. In Pattern Recognition (Proc. DAGM), volume 4174 of LNCS, pages 142-151, Berlin, Germany, September 2006. Springer.

38. T. B. Sebastian, P. N. Klein, and B. B. Kimia. On aligning curves. IEEE Transactions on Pattern Analysis and Machine Intelligence, 25(1):116-125, 2003.

39. Michael Spivak. A Comprehensive Introduction to Differential Geometry, Vol I 8 II. Publish or Perish, Inc., Berkeley, 1979.

40. S. Wang, Y. Wang, M. Jin, Xianfeng Gu, and Dimitris Samaras. 3D surface matching and recognition using conformal geometry. In CVPR (2), pages 2453-2460, 2006.

41. Y. Wang, M.-C. Chiang, and P. Thompson. Mutual information-based 3D surface matching with applications to face recognition and brain mapping. In International Conference on Computer Vision, 2005.

42. L. Wiskott, J.-M. Fellous, N. Krüger, and C. von der Malsburg. Face recognition by elastic bunch graph matching. In L. C. Jain, U. Halici, I. Hayashi, and S. B. Lee, editors, Intelligent Biometric Techniques in Fingerprint and Face Recognition, chapter 11, pages 355-396. CRC Press, 1999.

43. A-J. Yezzi and A. Mennucci. Conformal metrics and true "gradient flows" for curves. In Proceedings of the Tenth IEEE International Conference on Computer Vision, Volume 1, pages 913-919, 2005.

44. L. Younes. Computable elastic distance between shapes. SIAM Journal of Applied Mathematics, 58(2):565-586, 1998. 